\title{
Aneurysm of the Left Main Coronary Artery
}

\author{
Ênio E. Guérios, Ronaldo R. L. Bueno, Paulo M. P. Andrade, Deborah C. Nercolini, Álvaro L. A. \\ Pacheco, José C. E. Tarastchuk, Luiz F. Kubrusly \\ Curitiba, PR - Brazil
}

\begin{abstract}
Aneurysm of the left main coronary artery is a rare angiographic finding, with few cases described in the international literature. We report the case of a 42-year-old male with a previous history of acute myocardial infarction, whose coronariography indicated triple vessel coronary disease and an aneurysm of the left main coronary artery. A review of the etiology, clinical aspects, and surgical management of coronary arterial aneurysm is presented.
\end{abstract}

Aneurysms in coronary arteries are rare entities, however, with potential severe complications. Usually they are multiple and atherosclerosis is responsible for more than $50 \%$ of the diagnosed adult cases in the Western world. The most affected places are, in order of frequency, the, proximal and mid portions of the right coronary, the proximal portion of the anterior descending branch, and the proximal portion of the circumflex branch ${ }^{1,2}$. Aneurysm of the left coronary trunk is extremely infrequent, with little more than 30 cases described in the literature.

This report has the objective of presenting a case of aneurysm of the left main coronary artery and summarizing the available data from the literature about this uncommon entity and of its yet uncertain management.

\section{Case Report}

The patient was a 42-year-old male Caucasian fisherman with a 15-day history of acute myocardial infarction not treated with thrombolitics in his city of origin, as risk factors for coronary disease, smoking, and hyperlipemia where found. At cinecoronariography, occlusion of the right coronary, critical stenosis in the proximal portion of the anterior descending branch and circumflex branch, and an aneurysm of the left main coronary artery, measuring $23.6 \times 15.8 \mathrm{~mm}$, with stasis of contrast in its interior and an important delay in distal coronary flow (Fig.1) were documented. The left ventricle maintained adequate contractibility, with a $62 \%$ ejection fraction. With no findings in the clinical history or

Hospital Universitário Evangélico de Curitiba e Hospital Erasmo de Rotterdam - Curitiba

Mailing address: Ênio Eduardo Guérios - Rua Pedro Rolim de Moura, 25/502 80030-250 - Curitiba, PR - Brazil laboratory examinations that night suggest vasculitis or endocarditis, and with the basal disease related to aneurysm, atherosclerosis seemed to us to be the most probable cause.

The patient underwent myocardial revascularization, with the implant of the left internal mammary artery for the anterior descending and grafts of the saphenous vein for the first obtuse marginal branch and for the right coronary. The consensus as to the best strategy for treatment of aneurysm was to avoid the direct approach or closure of the aneurysm. The patient evolved well in postsurgery and was discharged from ITU on the second day. In the $180^{\circ}$ post operative, the patient remains asymptomatic with noninvasive tests negative for myocardial ischemia.

\section{Discussion}

Coronary aneurysm is defined as the presence of a segment of coronary artery with a diameter $\geq 1.5$ times the adjacent normal section (in the case of left main coronary artery $\geq 1.5$ times the diameter of the widest artery of the patient $)^{3,4}$, which may be fusiforme or saccular ${ }^{5}$. A variation in the incidence of aneurysm has been reported in the literature of between $0.15-4.9 \%$, among all patients whounderwent coronariography, with a strong predominance of males ${ }^{1,2}$. Such a discrepancy is probably due to the conceptual mix-up between aneurysm and coronary ectasia ${ }^{6}$. To differenciate the entities, Syed and Lesch ${ }^{4}$ classified a localized dilatation as aneurysm and ectasia as a more diffused dilatation, which affects at least half of the extension of artery ${ }^{4}$.

The first pathologic description of a coronary aneurysm was published by Morgagni in $1761^{7}$, and the first clinical case of an aneurysm was reported by Bourgon who, in 1812, described a postmortem finding of a dilatation in the right coronary in a patient who experienced sudden death ${ }^{8}$. Until 1967, all cases described in the literature were autopsy reports. The biggest series of aneurysms observed in coronariographies is from CASS (the Coronary Artery Surgery Study), which found that $4.9 \%$ of the total population of the register had aneurysmatic disease in the coronary arteries ${ }^{1}$. Evidence, however, exists that suggests that the incidence of ectasia in coronary arteries is increasing. Its etiology, treatment, and prognosis remain obscure, and knowledge about this entity should be improved ${ }^{9}$.

Potential causes of the formation of aneurysms in coronary arteries are cited in Table I. Kawasaki disease is the 
most common cause of coronary aneurysms in the world, and atherosclerotic coronary disease is the most common cause in the United States. Hartnell et al. ${ }^{3}$ in a prospective study of almost 5,000 cardiac catheterizations found coronary dilatations in 70 patients. Fifty-eight had significant coronary obstructions, while 12 did not.

The histologic study of these aneurysms reveals diffuse hyalinization, lipidic deposits, rupture of intima and medium calcification and focal fibrosis, and intramural hemorrhage $^{2}$. The presence of dilated segments in arteries with severe atherosclerotic disease or in arteries with structural alterations, as in Marfan Syndrome, supports the hypothesis that these ectasias are reflexes of evolvement and weakening of the medium layer of the vase. Aneurysms that are observed at the site of previous percutaneous coronary interventions also have their origin in damage of the vascular wall. Situations exist, however, in which the physiopathologic mechanisms that lead to the development of these dilatations has not yet been clarified.

Sorrell et al. ${ }^{9}$ suggest an association between the chronic stimulation of endogenous nitric oxide, with consequent chronic stimulation of vascular relaxation, and the occurrence of areas of ectasia in coronary arteries.

Whichever is the responsible mechanism, it is definite that the dilated sections present in coronary arteries are not benign entities. Reports in the literature ${ }^{10-12}$ show that these areas, even without the association of stenosis, are subject to spasms, thrombosis, and spontaneous dissection, and as such, are potential causes of acute myocardial infarction. Spasms, episodes of microembolization, and disturbances of blood flow caused by the presence of aneurysms may cause angina. In coronariography, flaws that occur in filling because of thrombus or because of inadequate flow of the contrast medium in the aneurysmatic region caused by dilution of the contrast with blood may lead to poor visualization of the arterial lumen and a flaw in the angiographic interpretation of the gravity of the associated coronary stenosis ${ }^{13}$.

Markis et al. ${ }^{14}$ were the first to prospectively evaluate patients with coronary ectasia. These authors described clinical aspects and evolution over 24 months of 30 patients with coronary ectasia found in a group of 2,500 patients, who consecutively underwent cardiac catheterization.

\begin{tabular}{|l|}
\hline Table I - Potential causes of formation of coronary \\
aneurysms
\end{tabular}

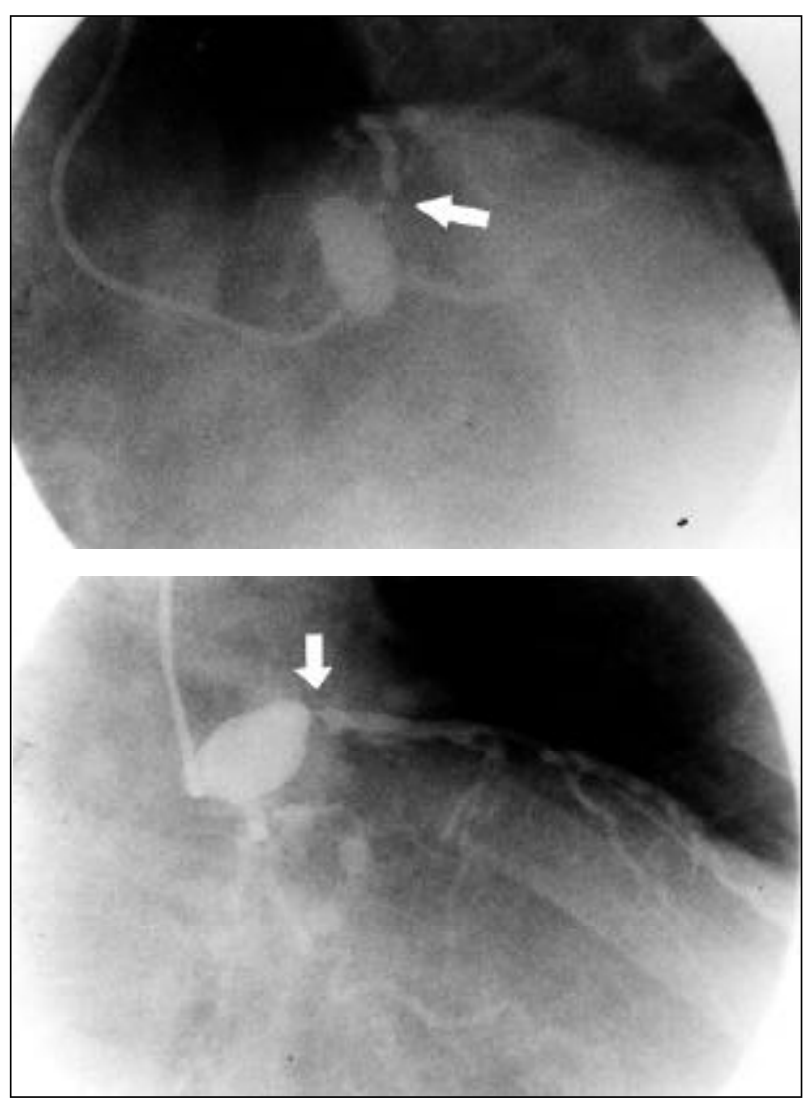

Fig. 1 - A) Left coronary in oblique anterior right. Arrow tip points to critical lesion in opening "ostium" of anterior descending branch; B) Left coronary in oblique anterior caudal left. Arrow tips point to critical lesions of descending anterior branch and circumflex branch.

They observed that these patients without obstructive coronary disease, but with ectasia, had a greater prevalence of systemic arterial hypertension, preceding infarction of the myocardium, and familial coronary disease history than the control group and had a $15 \%$ mortality in two years, equivalent to the percentage of mortality of patients with triple vessel coronary disease clinically treated at that time. On the contrary, in the previously mentioned study of Hartnell $\mathrm{el} \mathrm{al}^{3}$, among 500 evaluated patients, patients with coronary aneurysms evolved worse whether clinically or surgically treated when compared with patients without aneurys ms with the same degree of atherosclerotic coronary disease.

Apparently, however, the prognosis of patients with coronary aneurysm is primarily related to the severity of the concomitant obstructive coronary disease, with the exception of the few cases in which the dilatation of the vessel is so prominent and excentric that surgery is indicated, with resection of the aneurysm and arterioplasty, to prevent rupture ${ }^{1,15}$.

Only a few descriptions exist of surgically treated aneurysm of the left main coronary artery ${ }^{16}$. Some authors recommend distal closure of the dilated segment and concomitant revascularization of the descending anterior and circumflex artery, to prevent embolization or enlargement and possible rupture of the aneurysm ${ }^{16,17}$. Others suggest 
no closure, because of concern for the patency of the graft over mid- or long-term periods. Besides this, they maintain that, in cases of distal stenosis to aneurysm, a pressure gradient is formed and the presence of pervious bypass to the left coronary branches establishes a flow with retrograde direction to the aneurysm, preventing distal embolizations ${ }^{18,19}$.
Therefore, in this case, a direct approach or closure of the aneurysm was avoided. The risk of embolization is considered small-due to the expectation that the backward flow from the distal anastomosis of the grafts would exceed the forward flow through the critical lesions distal to the aneurysm (Fig. 1).

\section{References}

1. Swaye PS, Fisher LD, Litwin P, et al. Aneurysmal coronary artery disease. Circulation 1983; 67: 134-8.

2. Hawkins JW, Vacek JL, Smith GS. Massive aneurysm of the left main coronary artery. Am Heart J 1990; 119: 1406-8.

3. Hartnell GG, Parnell BM, Pridie RB. Coronary artery ectasia:its prevalence and clinical significance in 4993 patients. Br Heart J 1985; 54: 392-5.

4. Syed M, Lesch M. Coronary artery aneurysm: a review. Prog Cardiovasc Dis 1997; 40: 77-84.

5. Topaz O, DiSciascio G, Cowley MJ, et al. Angiographic features of left main coronary artery aneurysms. Am J Cardiol 1991; 67: 1139-42.

6. Araújo JAR, Cruz Jr A, Souza FJR. Aneurisma da artéria coronária direita. Rev Bras Cardiol Invas 1999; 7: 18-21.

7. Morgagni JB. De sedibus et causis morborum. Venetus Tom I, Epis 27, Art 28, 1761 citado por Ilia R, Goldfarb B, Gilutz H, Battler A. Aneurysm of the left main coronary artery: progression of dilatation with concomitant deterioration of coronary stenoses. Int J Cardiol 1994; 45: 135-7.

8. Bourgon A in Biblioth Med 1812; 37: 183, citado por Packard M, Weehsler HF. Aneurysm of the coronary arteries. Arch Int Med 1929; 43: 1-14.

9. Sorrell VL, Davis MJ, Bove AA. Current knowledge and significance of coronary artery ectasia:a chronologic review of the literature, recommendations for treatment, possible etiologies and future considerations. Clin Cardiol 1998; 21: $157-60$
10. Bove AA, Vlietstra RE. Spasm in ectatic coronary arteries. Mayo Clin Proc 1985; 60: 822-26.

11. Perlman PE, Ridgeway NA. Thrombosis and anticoagulation therapy in coronary ectasia. Clin Cardiol 1989; 12: 541-2.

12. Huikuri HV, Mallon SM, Myerburg RJ. Cardiac arrest due to spontaneous coronary artery dissection in a patient with with coronary ectasia - a case report. Angiology 1991;42: 148-51.

13. Wagdi $P$, Vuilliomenet A, Jenzer HR. Left main coronary aneurysm and severe three-vessel disease with sparing of na isolated conus artery. Am Heart J 1996; 131: 831-3.

14. Markis JE, Joffe CD, Cohn PF, Feen DJ, Herman MV, Gorlin R. Clinical significance of coronary artery ectasia. Am J Cardiol 1976; 37: 217-22.

15. Lazarus A, Donzeau-Gouge P, Spaulding C, Weber S, Guerin F. Surgical treatment of atherosclerotic aneurysm of the left main coronary artery. Am Heart $\mathrm{J}$ 1992; 123: 222-4.

16. Lepojarvi M, Salmela E, Huikuri H, Karkola P. Repair of na aneurysm of the left main coronary artery. Ann Thorac Surg 1996; 61: 1247-9.

17. Fukaya Y, Miyakawa M, Senga O, Hikita H, Kouzu S, Tunemoto H. Surgical management of left main coronary artery aneurysm. Ann Thorac Surg 1994; 57: 228-30.

18. Kitamura S. Invited commentary. Ann Thorac Surg 1990; 50: 293.

19. Olson JP, Chamusco RF. Giant left main coronary artery aneurysm initially seen as an acute coronary syndrome. Am Heart J 1995; 129: 1216-7. 